three HATCH categories. After a mean follow-up of $474 \pm 330$ days, the recurrence rate were $36.4 \%, 38.7 \%, 34.3 \%$, from $\mathrm{HATCH}=0$ to HATCH $\geq 2$ categories $(p=0.707)$. Univariate analysis revealed that nonparoxysmal AF, left atrium size, body mass index were predictors of AF recurrence. Multivariate analysis revealed that nonparoxysmal AF ( $\mathrm{HR}=1.43,95 \%$ CI 1.03 to $1.99, \mathrm{p}=0.031$ ) was the only independent predictor of AF recurrence. $\mathrm{HATCH}$ and left atrium size were not independent predictors of AF recurrence.

Conclusion HATCH has no value in prediction of AF recurrence after catheter ablation.

\section{Q0571 EFFECT OF VAGAL NERVE ON THE MONOPHASIC ACTION POTENTIAL OF VENTRICULAR OUTFLOW TRACT}

doi:10.1136/hrt.2010.208967.571

Chang Dong, Bai Xiaoyan, Zhang Shulong, Gao Lianjun, Yang Yanzong. First Affiliated Hospital of Dalian Medical University

Objective Vagal nerve may be related with idiopathetic ventricular tachycardia (IVT). The present study was aimed to investigate the effect of vagal nerve on the monophasic action potential (MAP) of ventricular outflow tract.

Methods Eight adult mongrel dogs were involved. Bilateral vagosympathetic tunks were decentralised for stimulation. Metoprolol was given to block sympathic effects. Three MAP recording electrode were placed at the left ventricular outflow tract (LVOT), right ventricular outflow tract (RVOT) and right ventricular apex (RVA) respectively through right femoral artery and vein. MAP was recorded at the LVOT, RVOT, RVA with or without vagal stimulation (VS) respectively.

Results MAP duration (MAPD) under VS was significantly shorter than baseline $(p>0.05)$. With or without VS, the MAPD at RVA were significantly shorter than that at RVOT and LVOT $(p<0.05)$, while there was no difference of MAPD between RVOT and LVOT. With VS, the abbreviation of MAPD at outflow tract was greater significantly than that at RVA (APD90 12.1 \pm 3.9 at RVOT, 14.8 \pm 5.5 at LVOT vs $8.3 \pm 4.1$ at RVA, $p<0.05)$, while there was no difference of MAPD between LVOT and RVOT ( $p>0.05$ ).

Conclusions VS could reduce MAPD significantly. With VS, the abbreviation of MAPD at outflow tract was greater significantly than that at RVA. It suggested that outflow tract may be sensible to vagal modulation, which might be related to the occurrence of IVT.

\section{e0572 COMPARISON OF PULMONARY VEINS OSTIUM, ANTRUM AND LEFT ATRIAL VOLUME IN PATIENTS WITH AND WITHOUT PAROXYSMAL ATRIAL FIBRILLATION}

doi:10.1136/hrt.2010.208967.572

Liu Min, Yang Yanzong, Zhang Shulong, Gao Lianjun. First Affiliated Hospital of Dalian Medical University

Objective To the date, catheter ablation basing on pulmonary vein (PVs) isolation is effective treatment for paroxysmal atrial fibrillation (PAF). In some experimental electrophysiological center, catheter ablation has been the first line for PAF. The aim of this study is to compare the variation of ostia, the antrum volume of pulmonary veins and the left atrial volume in patients with and without PAF. Methods We consecutively enrolled 28 and 35 patients with and without PAF (PAF group and control group). All the patients were taken intravenous injection of contrast medium before evaluated by 16-slice CT (MSCT). The 3D reconstruction of PVs and left atrium was transformed into AW4.2 system, and calculated the variation of pulmonary veins by Cardiac IQ software. Diameters of PVs ostia were measured by virtual endoscopy. The antrum volume of PVs and the left atrium volume were calculated by volume rendering. Results 3 patients $(10.7 \%)$ with PAF had common ostia or trunk of $\mathrm{PVs}$ and right middle PVs, respectively. We compared the maximum and minimum diameter of PVs ostia in PAF group with that in control group as follows: the maximum diameters, left superior PVs $(22.69 \pm 3.56$ vs $18.69 \pm 2.15 \mathrm{~mm}, \mathrm{p}<0.01)$, left inferior PVs $(18.40 \pm 2.50$ vs $16.96 \pm 2.07 \mathrm{~mm}, \mathrm{p}<0.05)$, right superior PVs $(20.78 \pm 3.46$ vs $19.26 \pm 2.55 \mathrm{~mm}, \quad \mathrm{p}=0.08)$, right inferior PVs $(20.19 \pm 4.39$ vs $16.90 \pm 1.75 \mathrm{~mm}, \mathrm{p}<0.01)$; the minimum diameter, left superior PVs $(16.18 \pm 3.60$ vs $11.12 \pm 2.55 \mathrm{~mm}, \mathrm{p}<0.01)$, left inferior PVs $(11.1 \pm 3.20$ vs $10.40 \pm 2.10 \mathrm{~mm}, \mathrm{p}<0.01)$, right superior PVs $(16.18 \pm 2.57$ vs $13.61 \pm 2.35 \mathrm{~mm}, \mathrm{p}<0.01)$, right inferior PVs $(16.26 \pm 3.16$ vs $13.29 \pm 2.20 \mathrm{~mm}, \mathrm{p}<0.01)$. The atrium volume of the bilateral PVs in PAF group was significantly larger than that in the control group (left, $3.53 \pm 0.74$ vs $2.74 \pm 0.49 \mathrm{~cm}^{3}, \quad \mathrm{p}<0.01$; right, $4.57 \pm 1.59$ vs $\left.3.54 \pm 1.01 \mathrm{~cm}^{3}, \mathrm{p}<0.01\right)$. The left atrial volume in PAF group was also significantly larger than that in control group ( $99.83 \pm 15.68$ vs $\left.88.24 \pm 18.21 \mathrm{~cm}^{3}, \mathrm{p}<0.05\right)$. The atrium volume of bilateral PVs justified with left atrial volume had no significant difference between the two groups.

Conclusion The ostial diameter and atrium volume of PVs were increased significantly, leading to the change for anatomy of atrial sleeves and distribution of autonomic nerve, and caused reentry or focal automaticity around PVs, which eventually initiated PAF. This study suggests that patients with PAF may exist potential structural disease of atria.

\section{E0573 IMPACT OF AGEING ON THE LEFT ATRIUM THROMBUS IN PATIENTS WITH NON-VALVULAR ATRIAL FIBRILLATION}

doi:10.1136/hrt.2010.208967.573

Tang Ri-bo, Liu Chang, Ma Chang-sheng, Dong Jian-zeng, Liu Xing-peng, Long De-yong, Yu Rong-hui. Beijing An Zhen Hospital

Objective This study sought to investigate the impact of ageing on left atrium thrombus in patients with non-valvular atrial fibrillation (AF). Methods Four hundred and twenty-one consecutive patients with AF underwent transesophageal echocardiography prior to AF ablation were included in a prospectively established database. The elderly group was defined as age more than 65 years old.

Results In all the 421 patients, 108 (25.6\%) were in the elderly group and $313(74.4 \%)$ were in the young group, with the mean age $70.0 \pm 3.7,52.1 \pm 8.5$, respectively. There was no significant difference of left atrium thrombus between the elderly group and the young group $(4.6 \%$ vs $6.7 \%, p=0.439)$. It was shown there is less value for age predicting left atrium thrombus, the area under the receptoroperating curve was 0.494 . The mean age $(56.7 \pm 9.9$ vs $56.7 \pm 10.9$, $\mathrm{p}=0.989)$ and the proportion of the elderly $(19.2 \%$ vs $26.1 \%$, $\mathrm{p}=0.439$ ) did not differ significantly between the patients with left atrium thrombus and those without. After adjustment for ejection fraction, heart failure, hypertension, diabetes mellitus, angiotensinconverting enzyme inhibitors/angiotensin receptor blockers medication in logistics multivariate analysis, left atrium diameter, non-paroxysmal AF, prior thromboembolic history were independent risk factors of left atrium thrombus. However, age was not an independent risk factor of left atrium thrombus.

Conclusions Ageing itself was not a risk factor of left atrium thrombus in patients with non-vavular AF.

\section{e0574 HIGH-NORMAL THYROID FUNCTION AND RISK OF RECURRENCE OF ATRIAL FIBRILLATION AFTER CATHETER ABLATION}

doi:10.1136/hrt.2010.208967.574

Tang Ribo, Liu Dongling, Dong Jianzeng, Liu Xingpeng, Long Deyong, Yu Ronghui, Ma Changsheng. Beijing An Zhen Hospital

Background It has been shown that serum free thyroxine (FT4) concentration is independently associated with atrial fibrillation (AF) even in euthyroid persons. This study aimed to testify the 
impact of high-normal level of FT4 on recurrence after catheter ablation of AF.

Methods Two hundred and forty-four consecutive patients with paroxysmal AF underwent circumferential pulmonary vein isolation (PVI) were prospectively enrolled. Exclusion criteria included prior or current thyroid dysfunction on admission, amiodarone medication for three months before admission.

Results After a mean follow-up of $416 \pm 204$ (91-856) days, the recurrence rates were $14.8 \%, 23.0 \%, 33.3 \%, 38.7 \%$ from the lowest FT4 quartile to the highest FT4 quartile, respectively $(\mathrm{p}=0.016)$. Adjustment for age, gender, left atrium diameter, PVI, there was an increased risk of recurrence in the subjects with the highest FT4 quartile compared with those with the lowest quartile (HR 3.31, $95 \%$ CI 1.45 to $7.54, p=0.004)$. As a continuous variable, FT4 was also an independent predictor of recurrence (HR 1.10, 95\% CI 1.02 to $1.18, \mathrm{p}=0.016$ ).

Conclusions Patients with high-normal thyroid function were at an increased risk of AF recurrence after catheter ablation.

\section{e0575 B-TYPE NATRIURETIC PEPTIDE ON CORONARY CIRCULATION IN YORK PIGS MODEL OF ACUTE MYOCARDIAL INFARCTION WITH HEART FAILURE}

doi:10.1136/hrt.2010.208967.575

${ }^{1}$ Jing Zhang, ${ }^{2}$ Xianghua Fu, ${ }^{1}$ Qingsheng Wang, ${ }^{1}$ Xinna Fan, ${ }^{2}$ Yanbo Wang, ${ }^{2}$ Xuechao Wang. ${ }^{1}$ The No. 1 Hospital of Qinhuangdao; ${ }^{2}$ The Second Hospital of Hebei Medical University

Objective To evaluate the impact of intravenous administration of rhBNP on coronary artery haemodynamics in York pigs model of AMI-HF.

Methods Total of 14 York pigs were included in this study. The AMIHF models was made by coronary occlusion and microthrombi perfusion, pigs were randmised into saline group and rhBNP group. Administration of rhBNP (bolus of $1.5 \mu \mathrm{g} / \mathrm{kg}$ followed by a continuous infusion of $0.01 \mu \mathrm{g} \cdot \mathrm{kg}^{-1} \cdot \mathrm{min}^{-1}$ for $60 \mathrm{~min}$, and then the dosage can be increased to $0.02-0.03 \mu \mathrm{g} \cdot \mathrm{kg}^{-1} \cdot \mathrm{min}^{-1}$ until LVEDP $<12 \mathrm{~mm}$ $\mathrm{Hg}$, maintaining $\mathrm{MBP} \geq 65 \mathrm{~mm} \mathrm{Hg}$ ) in rhBNP group. The saline group was given equal volume of normal saline using the same method. Coronary pressure $\left(\mathrm{P}_{\mathrm{c}}\right)$, the average peak velocity (APV), coronary vascular resistance (CR), coronary flow reserve (CFR) and coronary diameter were recorded simultaneously at baseline, instant after the model established, 60min after continuous infusion of $0.01 \mu \mathrm{g} \cdot \mathrm{kg}^{-1} \cdot \mathrm{min}^{-1} \mathrm{rhBNP}$ and the time point of LVEDP $<12 \mathrm{~mm} \mathrm{Hg}$. The coronary blood flow was measured at rest and maximal hyperaemia.

Results 12 animals achieved the standard of AMI-HF model successfully, the observation parameters were recorded at baseline before balloon occlusion, instant after the model established, $60 \mathrm{~min}$ after continuous infusion of $0.01 \mu \mathrm{g} \cdot \mathrm{kg}^{-1} \cdot \mathrm{min}^{-1} \mathrm{rhBNP}$ and the time point of LVEDP decreased to $<12 \mathrm{~mm} \mathrm{Hg}$. 1. Changes of Coronary artery parameters: There is no significant difference of coronary diameter, APV, CR and CFR in saline group. Coronary artery diameter increased after rhBNP administration. According to the intracoronary Doppler flow results, APV and CBF were significantly increased and CR decreased after rhBNP administration. CFR was significant rebound after continuous infusion of $0.01 \mu \mathrm{g} \cdot \mathrm{kg}^{-1} \cdot \mathrm{min}^{-1} \mathrm{rhBNP}$ for $30 \mathrm{~min}$. And compared with the control group at the same observation point, APV and CBF significantly increased and CR significantly decreased at the stage of infusion $0.010 \mu \mathrm{g} \cdot \mathrm{kg}^{-1} \cdot \mathrm{min}^{-1}$ Doppler parameters of renal artery, there was no difference of rhBNP. LVEF measured by echocardiography was lower than baseline after the models established and tended to increase after administration of rhBNP, but no significance was found compared with that in saline Group and that immediate after models established.
Conclusion It could increase blood flow of injury coronary artery, improve CFR and improve the coronary and administration of rhBNP in pigs with AMI-HF.

\section{Clinical and Research Medicine: Thrombosis e0576 CLINICAL ANALYSIS OF ANTICOAGULANT TREATMENT IN ACUTE PULMONARY EMBOLISM STRATIFIED AS INTERMEDIATE-RISK}

doi:10.1136/hrt.2010.208967.576

Wang Yong, Zhang Hongliang. Fu Wai Hospital

Objective To analyse the clinical effect of anticoagulant treatment in acute pulmonary embolism stratified as intermediate-risk.

Method Patients with intermediate-risk acute pulmonary embolism (PE) were enrolled in Center for Pulmonary Vascular Diseases, Fu Wai Hospital between Aug 2008 and Apr 2010. The intermediaterisk acute $\mathrm{PE}$ was defined as stable haemodynamics and the presence of right ventricular dysfunction (echocardiography or CT showed right ventricle dilation, pressure overload, BNP or NT-proBNP elevation) and/or markers of myocardial injury (stratified as intermediate-risk acute pulmonary embolism were included in this study. There were 29 men (42.6\%) and 39 women $(57.4 \%)$ with a mean age of $61.7 \pm 13.4$ years. Right ventricle (RV) dilatation or pressure overload was present on echocardiography or CT in 49 cases (\%), NT-proBNP elevation in 58 cases (\%) and cardiac troponin I positive in 8 cases (\%). The mean onset time was $15.7 \pm 18.0$ days. The main complaints included dyspnoea (60 patients, $88.2 \%$ ), chest pain (16 patinets, $23.5 \%$ ), cough (16 patients, $23.5 \%$ ), haemoptysis (8 patients, $11.8 \%)$, syncope (7 patients, 10.3\%), palpitation (5 patients, $7.4 \%$ ), dizziness (4 patients, $5.9 \%$ ) and cyanosis (1 patient, 1.5\%). Referring to ECG, 34 cases (50\%) presented S1OIIITIII and 27 cases (39.7\%) with T wave inversion in V1-V4 leads. The symptoms, physical signs and results of laboratory tests were improved significantly after anticoagulation by hemarin or low molecular weight heparin with a target INR of : heart rate $(82.5 \pm 14.8$ vs $69.9 \pm 7.5$ beats $/ \mathrm{min}, \quad \mathrm{p}<0.001)$ and $\mathrm{D}$-dimer (5.8 $\pm 4.7 \mathrm{mg} / \mathrm{l}$ vs $1.1 \pm 1.2 \mathrm{mg} / \mathrm{l}, \mathrm{p}<0.001 \mathrm{CTnT}$ or CTnI) positive. Results Sixty-eight patients significantly decreased; $\mathrm{PaO}_{2}$ $(68.7 \pm 11.7 \mathrm{~mm} \mathrm{Hg}$ vs $85.4 \pm 31.3 \mathrm{~mm} \mathrm{Hg}, \quad \mathrm{p}<0.001), \quad \mathrm{PCO}_{2}$ $(37.2 \pm 5.3 \mathrm{~mm} \mathrm{Hg}$ vs $40.3 \pm 4.6 \mathrm{~mm} \mathrm{Hg}, \quad \mathrm{p}<0.001)$ and $\mathrm{SaO}_{2}$ (93.5 $\pm 3.4 \%$ vs $95.6 \pm 2.1 \%, \mathrm{p}<0.001)$ significantly increased. During hospitalisation, ALT or AST was slightly increased in 12 cases (\%) and became normal after regular treatment; Twelve patients had mild bleeding, including 4 cases with positive urine occult blood, 4 cases with slight conjunctival haemorrhage, 2 cases with slight haemoptysis and 1 case with positive fecal occult blood.

Conclusions Anticoagulant treatment to patients with acute pulmonary embolism stratified as intermediate-risk significantly improved the symptoms, physical signs and results of laboratory tests with slight and low occurrence rate of complications.

\section{e0577 CLINICAL ANALYSIS OF FALSE NEGATIVE CASES BY VENTILATION-PERFUSION SCINTIGRAPHY COMPARED TO CT PULMONARY ANGIOGRAPHY IN DIAGNOSIS OF ACUTE PULMONARY EMBOLISM}

doi:10.1136/hrt.2010.208967.577

Wang Yong, Zhang Hongliang. Fu Wai Hospital

Objective Although ventilation-perfusion scintigraphy (V/O scan) is a robust and well established diagnostic test for suspected pulmonary embolism, false negative cases still exist. This study 